\title{
SOME REMARKS ON THE THEORY OF THE DIELECTRIC CONSTANT OF NON-POLAR DENSE GASES
}

\author{
B. R.A. NIJBOER \\ Instituut voor Theoretische Fysica, Rijksuniversiteit Utrecht, \\ Utrecht, The Netherlands
}

Received 18 February 1975

It is pointed out that the usual simple theory of the dielectric constant in isotropic nonpolar media, based on a model with constant polarizability and with dipolar interaction between atoms, is consistent only if the atoms are assumed to have a hard core, so that they cannot approach each other too closely.

The expansion of the dielectric constant in powers of the polarizability $\alpha$ is obtained in a simple way. The two-particle contribution to the deviation $S(\alpha, Q, T)$ from the law of Clausius-Mossotti is summed to all orders in $\alpha$ and in particular the term of $S(\alpha, Q, T)$ linear in the density $\varrho$ is discussed.

\section{Introduction}

We consider a uniform and isotropic dense gas consisting of $N$ neutral spherical atoms placed in a static external electric field $e_{0}(\boldsymbol{r})$. The atoms are supposed to interact with induced dipole forces only and for simplicity we further assume that the atoms have a constant (i.e. independent of the instantaneous configuration of the surrounding atoms) static polarizability $\alpha$.

In the statistical theory of the dielectric constant in nonpolar media as initiated by Kirkwood ${ }^{1}$ ) and by Yvon $^{2}$ ) and since then investigated and extended by many authors ${ }^{3}$ ) one usually starts from the following equations for the induced dipole moments $\boldsymbol{\mu}_{i}$ (the indices $i, k, \ldots$ number the atoms)

$$
\mu_{i}=\alpha e_{0}(i)-\alpha \sum_{k} T_{i k} \cdot \mu_{k}
$$

Here the dipolar tensor $\mathrm{T}_{i k}$ is defined by

$$
\mathrm{T}_{i k}=\nabla_{i} \nabla_{k} \frac{1}{r_{i k}}=\frac{1}{r_{i k}^{3}}\left(1-3 \frac{r_{i k} r_{i k}}{r_{i k}^{2}}\right), \quad \text { if } \quad i \neq k ; \quad \mathrm{T}_{i i}=0
$$


where $\boldsymbol{r}_{i k}=\boldsymbol{r}_{k}-\boldsymbol{r}_{i}, \boldsymbol{r}_{k}$ and $\boldsymbol{r}_{i}$ denote the positions of atoms $k$ and $i$. If we introduce the $3 N \times 3 N$ symmetric matrix

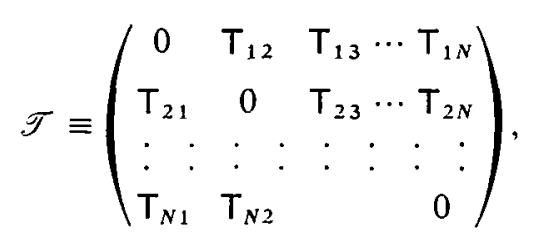

with eigenvalues $\lambda_{v}\left(\boldsymbol{r}_{1}, \boldsymbol{r}_{2}, \ldots, \boldsymbol{r}_{N}\right),(v=1,2, \ldots, 3 N)$, we may in obvious $3 N$ dimensional notation rewrite eqs. (1) as

$$
(1+\alpha \mathscr{T}) \cdot \boldsymbol{\mu}=\alpha \boldsymbol{e}_{0}
$$

From this we infer that eqs. (1) have a unique solution for the induced dipole moments $\mu_{i}$ as long as

$$
\operatorname{det}|1+\alpha \mathscr{T}|=\prod_{v}\left(1+\alpha \lambda_{v}\right) \neq 0 .
$$

If, however, for some value of the index $v$

$$
1+\alpha \lambda_{v}=0
$$

then, as will be evident from (5), eqs. (1) are either dependent or incompatible.

On the other hand, let us consider for a moment the homogeneous equations

$$
(1+\alpha \mathscr{T}) \cdot \boldsymbol{\mu}=0,
$$

i.e., we take the external field equal to zero. If now relation (6) holds for some $v$, then eqs. (7) have a nontrivial solution, that is, we have a case of spontaneous polarization. In the special case of the Drude-Lorentz atomic model, that is, when the atoms are represented by isotropic harmonic oscillators of eigenfrequency $\omega_{0}$ which again interact with dipole-dipole forces (a problem which in different context has been treated extensively in a previous publication ${ }^{4}$ ), it is easily shown that the eigenfrequencies of the coupled-oscillator system are given by

$$
\Omega_{v}=\omega_{0}\left(1+\alpha \lambda_{v}\right)^{\frac{1}{2}} .
$$

The condition (6) in this case leads to a frequency zero, while for a negative value of $1+\alpha \lambda_{v}$ (for some $v$ ) the oscillator model is seen to break down.

In view of the situation discussed above one may ask: what are the eigenvalues $\lambda_{v}$ of the matrix $\mathscr{T}$ ? For an arbitrary configuration of $N$ atoms it is very difficult to answer this question. However, let us discuss some simple cases. For the special 
case of an ideal fcc crystal Lucas ${ }^{5}$ ) has numerically computed the $\lambda_{v}$ (as a consequence of the periodicity of the crystal the problem reduces to a determination of the eigenvalues of a $3 \times 3$ matrix for every value of a wave vector $\boldsymbol{k}$ ). The smallest $\lambda_{v}$ turns out to be $-\frac{4}{3} \pi \varrho$, where $\varrho$ is the number density. This would apply to all cubic crystals. We see therefore that

$$
\operatorname{det}|1+\alpha \mathscr{T}| \neq 0,
$$

if only $4 \pi \varrho \alpha<3$. For crystals of the noble gases like $\operatorname{Ar}(4 \pi \varrho \alpha=0.5196)$ and $\mathrm{Xe}(4 \pi \circ \alpha=0.8372)$ this inequality is amply satisfied.

For a system of two particles $(N=2)$ at distance $r$, the 6 values of $\lambda_{v}$ are easily seen to be $\pm 2 / r^{3}, \pm 1 / r^{3}, \pm 1 / r^{3}$. For this simple system condition (9) will certainly be fulfilled as long as $r^{3}>2 \alpha$. For a cluster of three particles (at equal distance $r$ ) we find that a sufficient condition is $r^{3}>\frac{5}{2} \alpha$ and the same inequality is found for a cluster of four particles arranged in the vertices of a regular tetrahedron. For more particles the determination of the eigenvalues $\lambda_{v}$ seems rather difficult, but we think it rather plausible that the theory of the dielectric constant, based on the model discussed above, is consistent as long as the distance of closest approach between atoms is larger than say $2 \alpha^{1 / 3}$. Thus the atoms effectively should have a hard core with a diameter not smaller than this value. This restriction to the applicability of the theory is not too severe: for instance in the case of argon $\alpha \sigma^{-3}$ is of the order of 0.04 , where $\sigma$ (from the Lennard-Jones interaction $q(r)=4 \varepsilon$ $\left.\times\left[(\sigma / r)^{12}-(\sigma / r)^{6}\right]\right)$ is the distance where the potential of interaction has the value 0 . Hence the requirement that the distance of closest approach should be larger than $2 \alpha^{1 / 3}$ in this case would mean that the hard-core diameter should be larger than $0.7 \sigma$. We shall return to this point below.

\section{The expansion formula for the dielectric constant}

In order to find an expression for the static dielectric constant $\varepsilon$ of a nonpolar medium and in particular to study the deviations from the well-known law of Clausius-Mossotti

$$
(\varepsilon-1) /(\varepsilon+2)=\frac{4}{3} \pi \varrho \alpha,
$$

one usually assumes the existence of a shape-independent dielectric constant, defined by

$$
\boldsymbol{P}=[(\varepsilon-1) / 4 \pi] \boldsymbol{E},
$$

(e.g. $c f$. for a discussion of this point Ramshaw $\left.{ }^{3}\right)$ ). The fundamental equations (1) are then solved by iteration for a conveniently chosen macroscopic system of atoms 
and the resulting expression for the induced dipole moment $\boldsymbol{\mu}_{i}$ is averaged over microscopic configurations to obtain the polarization density $\boldsymbol{P}$. Obviously it is convenient to choose the macroscopic system in such a way that the external field $\boldsymbol{e}_{0}$ and the polarization $\boldsymbol{P}$ are uniform in the medium.

For instance if one considers a slab of dielectric material between the plates of a plane condensor, where $\boldsymbol{e}_{0}(i)=\boldsymbol{D}=\varepsilon \boldsymbol{E}$ for any $i$, one finds from (1) by iteration:

$$
\begin{aligned}
(\varepsilon-1) / \varepsilon= & 4 \pi, a \alpha u \cdot\left(1-\alpha \sum_{k}\left\langle\mathrm{~T}_{i k}\right\rangle+\alpha^{2} \sum_{k, l}\left\langle\mathrm{~T}_{i k} \cdot \mathrm{T}_{k l}\right\rangle\right. \\
& \left.-\alpha^{3} \sum_{k, l, m}\left\langle\mathrm{~T}_{i k} \cdot \mathrm{T}_{k l} \cdot \mathrm{T}_{l m}\right\rangle \cdots\right) \cdot u
\end{aligned}
$$

where $\boldsymbol{u}$ is a unit vector perpendicular to the plates, the brackets indicate an average over configurations and the summations (which are shape-dependent) are to be extended over the slab. Kirkwood $\left.{ }^{1}\right)$ shows that $\sum_{k}\left\langle\mathrm{~T}_{i k}\right\rangle=4 \pi \varrho\left(\boldsymbol{u} u-\frac{1}{3} 1\right)$.

By introducing the auxiliary quantity

$$
\boldsymbol{u}_{0}=\alpha \boldsymbol{D}-\alpha \sum_{k}\left\langle\mathrm{~T}_{i k}\right\rangle \cdot\left\langle\boldsymbol{\mu}_{k}\right\rangle=\frac{1}{3}(\varepsilon+2) \alpha E,
$$

De Boer, Van der Maesen and Ten Seldam ${ }^{6}$ ) rewrite (12) in the form

$$
\begin{aligned}
& (\varepsilon-1) /(\varepsilon+2) \\
& \quad=\frac{4}{3} \pi \varrho \alpha \boldsymbol{u} \cdot\left(1+\alpha^{2} \sum_{k l}\left(\left\langle\mathrm{~T}_{i k} \cdot \mathrm{T}_{k l}\right\rangle-\left\langle\mathrm{T}_{i k}\right\rangle \cdot\left\langle\mathrm{T}_{k l}\right\rangle\right)+\mathcal{O}\left(\alpha^{3}\right)\right) \cdot \boldsymbol{u},
\end{aligned}
$$

which directly leads to an expression for the deviation $S(\alpha, \varrho, T)$ from the ClausiusMossotti result defined by

$$
(\varepsilon-1) /(\varepsilon+2)=\frac{4}{3} \pi \varrho \alpha[1+S(\alpha, \varrho, T)]
$$

However, it is perhaps even simpler to consider a dielectric sphere in a homogeneous external field $e_{0}$. From macroscopic electrostatics one knows that in this case the electric field inside the sphere $E=[3 /(\varepsilon+2)] e_{0}$ and hence iteration of eqs. (1) immediately gives:

$$
\begin{aligned}
(\varepsilon-1) /(\varepsilon+2)= & \frac{4}{3} \pi \varrho \alpha u \cdot\left(1-\alpha \sum_{k}\left\langle\mathrm{~T}_{i k}\right\rangle+\alpha^{2} \sum_{k l}\left\langle\mathrm{~T}_{i k} \cdot \mathrm{T}_{k l}\right\rangle\right. \\
& \left.-\alpha^{3} \sum_{k l m}\left\langle\mathrm{~T}_{i k} \cdot \mathrm{T}_{k l} \cdot \mathrm{T}_{l m}\right\rangle \cdots\right) \cdot \boldsymbol{u},
\end{aligned}
$$

where $u$ again is a unit vector in the direction of the external field $e_{0}$, but the summations are now extended over a spherical volume. The position of atom $i$ may be taken in the centre of the sphere. One easily verifies that now $\sum_{k}\left\langle T_{i k}\right\rangle=0$ 
(even if $i$ is not in the centre). For the deviation from CM we now find

$$
S(\alpha, \varrho, T)=\boldsymbol{u} \cdot\left(\alpha^{2} \sum_{k l}\left\langle\mathrm{~T}_{i k} \cdot \mathrm{T}_{k l}\right\rangle-\alpha^{3} \sum_{k l m}\left\langle\mathrm{~T}_{i k} \cdot \mathrm{T}_{k l} \cdot \mathrm{T}_{l m}\right\rangle+\cdots\right) \cdot \boldsymbol{u} .
$$

Because of the spherical symmetry of the system this may be written as

$$
S(\alpha, \varrho, T)=\frac{1}{3} \operatorname{Tr}\left(\alpha^{2} \sum_{k l}\left\langle\mathrm{~T}_{i k} \cdot \mathrm{T}_{k l}\right\rangle-\alpha^{3} \sum_{k l m}\left\langle\mathrm{~T}_{i k} \cdot \mathrm{T}_{k l} \cdot \mathrm{T}_{l m}\right\rangle+\cdots\right),
$$

where again the summations are over a macroscopic spherical volume. Eqs. (14) and (18) represent equivalent expansions of the function $S$ in powers of $\alpha$. One notices (and this has been pointed out before by several authors) that, because of the fact that indices in different $T$ factors may well be equal, the coefficient of the term $\alpha^{n}$ has contributions from $2,3, \ldots,(n+1)$ particles. For instance an exact evaluation of the $a^{2}$ term would require knowledge of the 3-particle, as well as of the 2-particle molecular distribution function.

De Boer et al. ${ }^{6}$ ) have approximately evaluated the $\alpha^{2}$ term in the case of a Lennard-Jones interaction between the atoms by expanding it into powers of the density $\varrho$ and computing the terms $\varrho$ and $\varrho^{2}$ numerically. The 2 - and 3-particle distribution functions are accurately known to this order in the density. It turned out that the theoretical result calculated in this way is in qualitative agreement with experimental results for the function $S$ obtained by Michels $e t$ al. ${ }^{7}$ ) for compressed argon gas. Quantitatively there are deviations, for instance the theoretical slope of the function $S(\alpha, \varrho, T)$ plotted as a function of $\varrho$ is at the origin $(\varrho=0)$ roughly half the measured value.

\section{The function $S(\alpha, \varrho, T)$ to lowest order in the density}

In this section I want to point out that the 2-particle contribution [which I shall call $S^{(2)}(a, \varrho, T)$ ] to the function $S$ as given by the infinite expansion (18) can be summed exactly and expressed in terms of the radial distribution function $g(r)$. Indeed we have:

$$
S^{(2)}(\alpha, \varrho, T)=\frac{1}{3} \operatorname{Tr}\left(a^{2} \sum_{k}\left\langle\dot{T}_{i k} \cdot T_{k i}\right\rangle-a^{3} \sum_{k}\left\langle T_{i k} \cdot T_{k l} \cdot T_{i k}\right\rangle+\cdots\right) \cdot
$$

Now the eigenvalues of the tensor $T(r)$ are evidently $-2 / r^{3}, 1 / r^{3}, 1 / r^{3}$ and hence it follows that

$$
\begin{aligned}
S^{(2)}(\alpha, \varrho, T) & =\frac{1}{3} \varrho \int g(r) \sum_{n=2}^{\infty}\left(-\alpha / r^{3}\right)^{n}\left[2.1^{n}+(-2)^{n}\right] \mathrm{d}^{3} r \\
& =8 \pi \varrho \alpha^{2} \int_{0}^{\infty} \frac{g(r)}{r^{4}} \frac{\mathrm{d} r}{\left(1+\alpha / r^{3}\right)\left(1-2 \alpha / r^{3}\right)} .
\end{aligned}
$$


As might have been anticipated from the discussion in the introduction, we again observe here that this result is finite only if the distance of closest approach between atoms (which we shall call $\sigma_{0}$ ) is such that $\sigma_{0}^{3}>2 \alpha$.

To lowest order in $\varrho$ the radial distribution function $g(r)$ is given by

$$
g(r) \simeq \mathrm{e}^{-\beta \varphi(r)},
$$

where $\beta=(k T)^{-1}$ and $\varphi(r)$ is the potential of interaction between atoms. If we take for $\varphi(r)$ a Lennard-Jones potential cut off so as to give a hard core with diameter $\sigma_{0}$

$$
\varphi(r)=\left[\begin{array}{ccc}
4 \varepsilon\left[(\sigma / r)^{12}-(\sigma / r)^{6}\right], & \text { for } & r>\sigma_{0}, \\
\infty, & \text { for } & r<\sigma_{0},
\end{array}\right.
$$

the term linear in $\varrho$ of the function $S^{(2)}$ (and also of $S$ ) is given by

$$
\frac{8 \pi \rho \alpha^{2}}{3} \int_{0}^{\sigma_{0}^{1 / 3}} \exp \left[-4 \beta \varepsilon \sigma^{6}\left(\sigma^{6} x^{4}-x^{2}\right)\right] \frac{\mathrm{d} x}{(1+\alpha x)(1-2 \alpha x)},
$$

where $x=r^{-3}$ has been introduced as a new variable.

As was mentioned above in the case of argon $\alpha \sigma^{-3} \sim 0.04$. It follows that in order to avoid the singularity in the integrand we should require in this case that $\sigma_{0} \gtrsim 0.43 \sigma$. If we adhere to the stronger condition mentioned in the introduction that $\sigma_{0}>2 \alpha^{\mathrm{t} / 3}$ we should require $\sigma_{0} \gtrsim 0.68 \sigma$. On the other hand for $\beta \varepsilon \sim 1$ and $x \gtrsim 1.5 \sigma^{-3}$ the exponential in (23) is completely negligible, that is, for $r \lesssim(1.5)^{-1 / 3} \sigma$ $=0.87 \sigma$. Hence there is an appreciable range of values for the hard-core diameter $\sigma_{0}\left(0.43 \sigma \lesssim \sigma_{0} \lesssim 0.87 \sigma\right)$, for which the singularity is avoided and the integral is independent of $\sigma_{0}$. For such values of $\sigma_{0}$ we may write for (23)

$$
\frac{8}{3} \pi \varrho \alpha^{2} \int_{0}^{\infty} \exp \left[-4 \beta \varepsilon \sigma^{6}\left(\sigma^{6} x^{4}-x^{2}\right)\right]\left(1+\alpha x+3 \alpha^{2} x^{2}+\cdots\right) \mathrm{d} x .
$$

The terms $\alpha x$ and $3 \alpha^{2} x^{2}$ in (24) give a correction to the term linear in $\varrho$ of the function $S$ as calculated by De Boer et $a l^{6}$ ). We estimate that this correction (which is positive) in the case of argon amounts to a few percent only.

We should remind the reader here that Mazur and Jansen ${ }^{8}$ ) have extended the model discussed in this note in that they have taken into account the circumstance that the polarizability of an atom in a dense medium is different from that of a free atom and that this difference depends on the configuration of surrounding atoms. It is only for the harmonic-oscillator model of Drude-Lorentz that this effect does not occur. According to their estimate this effect gives rise to a contribution to the term linear in $\varrho$ of the function $S$ of the same order of magnitude as that calculated by De Boer et al. (and slightly modified above). 
Further I want to remark that, as I discovered recently, the result (20) has essentially been given (but derived in a quite different way) already by Isihara ${ }^{9}$ ), except that it erroneously contains a term linear in $\alpha$.

Finally, it may be noted that similar remarks as presented here with regard to the theory of the static dielectric constant might be made concerning the theory of the frequency-dependent dielectric constant (or of the refractive index).

\section{References}

1) J. G. Kirkwood, J. chem. Phys. 4 (1936) 592.

2) J.Yvon, Recherches sur la Théorie Cinétique des Liquides (Hermann, Paris, 1937).

3) $C f$. for a review of the older literature:

W.F. Brown Jr., Handbuch der Physik, S. Flügge, ed. (Springer, Berlin, 1956), vol. XVII, p.1. For more recent references, e.g.:

J. D. Ramshaw, Physica 62 (1972) 1.

D. Bedeaux and P.Mazur, Physica 67 (1973) 23.

4) B. R. A. Nijboer, Physica 79A (1975) 420.

5) A. Lucas, Physica 35 (1967) 353.

6) J.de Boer, F.van der Maesen and C.A.ten Seldam, Physica 19 (1953) 265.

7) A. Michels, C.A.ten Seldam and S. D.J.Overdijk, Physica 17 (1951) 781.

A. Michels and A. Botzen, Physica 15 (1949) 769.

8) P. Mazur and L. Jansen, Physica 21 (1955) 208.

9) A. Isihara, J. chem. Phys. 38 (1963) 2437. 